\title{
A importância dos indicadores biomédicos no funcionamento sexual em adultos portugueses saudáveis
}

\author{
Paula Teixeira ${ }^{a, b, *}$, Henrique Pereira ${ }^{a, b, c}$, Samuel Monteiro ${ }^{a, b}$, Graça Esgalhado ${ }^{a, b}$, \\ Rosa Marina Afonso ${ }^{a, b}$, Manuel Loureiro ${ }^{a, b}$, Delfina Ferrão ${ }^{d}$ y Nuno Garcia ${ }^{a, e}$
}

\author{
a Universidade da Beira Interior, Covilhã, Portugal \\ b Universidade da Beira Interior, Departamento de Psicologia e Educação, Covilhã, Portugal \\ c Universidade da Beira Interior, Centro de Investigação em Ciências da Saúde (CICS), Covilhã, Portugal \\ d Agrupamento de Centros de Saúde Cova da Beira, Centro de Saúde da Covilhã, Covilhã, Portugal \\ e Universidade da Beira Interior, Departamento de Informática, Covilhã, Portugal
}

Recibido el 19 de julio de 2017; aceptado el 30 de diciembre de 2017

\author{
PALAVRAS-CHAVE \\ Funcionamento \\ sexual; \\ Indicadores \\ biomédicos; \\ Sexualidade
}

\begin{abstract}
Resumo
Introdução: Na presente investigação pretendeu-se analisar a influência de indicadores biomédicos, tais como o índice de massa corporal, glicémia, colesterol total, triglicerídeos, pressão arterial sistólica e pressão arterial diastólica, no funcionamento sexual em adultos portugueses. Métodos: A amostra foi constituída por 225 indivíduos saudáveis (não fumadores, mulheres não tomando pílula contracetiva), entre os 18 e os 89 anos (média $=41$ ), 107 do sexo masculino e 117 do feminino. Aplicaram-se um questionário sociodemográfico, a versão portuguesa do Massachusetts General Hospital Sexual Functioning Questionnaire (MGH-SFQ), e dispositivos de medida de indicadores biomédicos.

Resultados: Dos participantes, 59,6\% ( $\mathrm{n}=134)$ apresentaram níveis para o funcionamento sexual global abaixo dos valores esperados, os homens pontuaram melhor funcionamento sexual global quando comparados com as mulheres, assim como o grupo dos participantes mais novos. Destacam-se os níveis de correlação significativa entre o IMC ( $r=-0,253 ; p<0,001)$, a glicémia $(r=-0,230 ; p<0,001)$, o colesterol total $(r=-0,144 ; p<0,05)$ e o funcionamento sexual. 0 modelo de regressão hierárquica permitiu demonstrar o efeito mediador das variáveis biomédicas sobre o funcionamento sexual, explicou $31 \%\left(r^{2}=0,31 ; p<0,001\right)$ da variância total.
\end{abstract}

\footnotetext{
* Autor para correspondência.

Correo electrónico: hpereira@ubi.pt (P. Teixeira).
} 


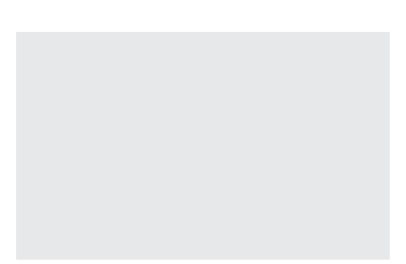

\section{KEYWORDS}

Sexual functioning;

Biomedical

indicators;

Sexuality
Discussão e conclusões: Esta investigação permite evidenciar a importância dos indicadores biomédicos no funcionamento sexual, ajuda os técnicos que trabalhem nessa área a estarem atentos ao modo como os contextos biomédicos interferem na expressão sexual.

(C) 2018 Asociación Española de Andrología, Medicina Sexual y Reproductiva. Publicado por Elsevier España, S.L.U. Todos los derechos reservados.

The importance of biomedical indicators in sexual functioning in healthy Portuguese adults

\begin{abstract}
Background: The aim of this study was to investigate the influence of biomedical indicators, such as body mass index, blood glucose, total cholesterol, triglycerides, systolic blood pressure and diastolic blood pressure, on sexual functioning in Portuguese adults.

Methods: The sample consisted of 225 healthy individuals (non-smokers, women not taking contraceptive pill), aged 18-89 years (mean $=41$ years), of whom 107 were males and 117 females. A Sociodemographic Questionnaire, the Portuguese version of the Massachusetts General Hospital Sexual Functioning Questionnaire (MGH-SFQ) and biomedical indicators measurement devices were applied.

Results: $59.6 \%(\mathrm{~N}=134)$ of the participants presented levels for global sexual functioning below the expected values. Men scored better overall sexual functioning when compared to women, as well as the group of younger participants. Significant correlational values between BMI $(r=-253, p<.001)$, glycemic index $(r=-.230, p<.001)$, total cholesterol $(r=-144, p<.05)$ and sexual functioning were obtained. The hierarchical regression model allowed to demonstrate the mediating effect of the biomedical variables on sexual functioning, explaining $31 \%$ $(\mathrm{r} 2=.31 ; \mathrm{p}<.001)$ of the total variance.

Discussion and conclusions: This research makes it possible to highlight the importance of biomedical indicators in sexual functioning, helping professionals working in this area to be aware of how biomedical contexts interfere with sexual expression.

(C) 2018 Asociación Española de Andrología, Medicina Sexual y Reproductiva. Published by Elsevier España, S.L.U. All rights reserved.
\end{abstract}

\section{Introdução}

A experiência humana integra diversos processos. A função sexual é um deles e está associada a relacionamentos íntimos e prazerosos, assim como à qualidade de vida ${ }^{1,2}$. Quando os mecanismos sexuais que estão na base da resposta sexual humana ${ }^{3}$ estão de certa forma condicionados, podemos falar de disfunção sexual.

Pode-se afirmar que as disfunções sexuais constituem um problema que ainda é bastante prevalente e de grande impacto na população mundial ${ }^{4}$, podem surgir durante diferentes fases do ciclo de resposta sexual, nos homens é de $20 \%$ a $30 \%$, enquanto que nas mulheres é de $30 \%$ a $45 \%^{5-11}$. Esses dados são congruentes com as estimativas obtidas na população portuguesa ${ }^{12}$.

A experiência de disfunção sexual pode ser influenciada quer por variáveis psicoemocionais, quer por variáveis biomédicas. Neste trabalho, enfatiza-se a importância de variáveis biomédicas, há já alguma literatura que corrobora a interferência dessas no funcionamento sexual. Por exemplo, disfunção sexual está diretamente relacionada com o índice de massa corporal elevado ${ }^{13}$, com a diabetes ${ }^{13,14}$, níveis de triglicéridos e colesterol elevados ${ }^{15,16}$, doenças cardiovasculares ${ }^{17,18}$ e hipertensão arterial ${ }^{19}$.
Na medida em que não existe em Portugal um estudo sobre esse tema, fez-se a presente investigação, cujo objetivo é determinar a relação preditiva, por meio de um modelo de regressão múltipla hierárquica, da influência dos indicadores biomédicos no funcionamento sexual. A partir desse objetivo geral estabeleceram-se outros mais específicos, tais como comparar a diferença dos níveis de funcionamento sexual entre homens e mulheres e comparar a diferença dos níveis de funcionamento sexual entre pessoas mais novas e pessoas mais velhas.

De acordo com os estudos revistos, espera-se encontrar altas prevalências de disfunção sexual no género feminino, quando comparado com o género masculino. Também é de esperar que os jovens tenham melhor funcionamento sexual quando comparados com a população mais velha. Presumese que exista relação entre algumas variáveis biomédicas, como é o caso da pressão arterial, glicémia e IMC, e o funcionamento sexual.

Posto isso, fizeram-se as seguintes relações hipotetizadas que se vai procurar testar no âmbito desta investigação e que integram o cerne do presente trabalho: são preditores de melhor funcionamento sexual níveis normativos dos indicadores biomédicos; os homens têm melhor funcionamento sexual do que as mulheres; as pessoas mais novas 
têm melhor funcionamento sexual do que as pessoas mais velhas.

\section{Método}

\section{Participantes}

A amostra usada é constituída por 225 indivíduos saudáveis (foram excluídos indivíduos fumadores e mulheres que tomam pílula). Dos participantes, 107 (47,80\%) são homens e 117 (52,20\%) são mulheres, com uma média de aproximadamente 41 anos e o desvio-padrão de 18,46 (M = 40,53; $\mathrm{DP}=18,46)$. Relativamente ao estado marital, $74(33,20 \%)$ são casados, $69(30,90 \%)$ são solteiros e $37(16,60 \%)$ referem estar a namorar ou terem um compromisso afetivo. Além disso, $18(8,10 \%)$ estão em união de facto, $16(7,20 \%)$ estão divorciados ou separados e nove $(4,00 \%)$ estão viúvos. Em relação ao local de residência é de constatar que a maior parte dos participantes habita numa pequena cidade $(169,75,40 \%), 28(12,50 \%)$ habitam numa grande cidade, 19 $(8,50 \%)$ num pequeno meio rural e apenas oito $(3,60 \%)$ num grande meio rural. Ao nível da escolaridade é de salientar que $82(38,70 \%)$ têm até 12 anos, 65 (30,70\%) têm licenciatura ou bacharelato, $34(16,00 \%)$ até nove anos de escolaridade e 31 (14,60\%) têm mestrado ou doutoramento. Quando perguntados sobre a sua situação profissional, 71 $(31,80 \%)$ afirmaram ser estudantes, 68 (30,50\%) trabalhadores por conta de outrem, $27(12,10 \%)$ reformados, $25(11,20 \%)$ trabalhadores por conta própria, $23(10,30 \%)$ desempregados e nove $(4,00 \%)$ trabalhadores-estudantes. No que se refere ao estatuto socioeconómico salienta-se que $111(49,80 \%)$ afirmam ter médio, $72(32,30 \%)$ baixo-médio, $22(9,90 \%)$ médio-alto, $15(6,70 \%)$ baixo e apenas três $(1,30 \%)$ alto. Por último, quando inquiridos sobre a sua orientação sexual, a grande maioria autoidentificou-se como heterossexual (196, 87,50\%), $20(8,90 \%)$ autoidentificaram-se como homossexuais e oito $(3,60 \%)$ como bissexuais.

\section{Instrumentos}

Para a concretização dos objetivos da presente investigação usou-se um questionário sociodemográfico elaborado por nós de modo a abranger um conjunto de questões que nos possibilitam o levantamento de informação geral dos participantes. Nele reunimos um conjunto de questões com o intuito de recolher informação pertinente para a caracterização da amostra, tais como idade, género sexual, escolaridade, situação profissional, estatuto socioeconómico e orientação sexual.

Para se avaliar o funcionamento sexual usou-se o Massachusetts General Hospital - Sexual Functioning Questionnaire $(\mathrm{MGH}-\mathrm{SFQ})^{20}$, na versão portuguesa validada psicometricamente ${ }^{21}$. O MGH-SFQ é constituído por uma escala com cinco itens que avaliam o interesse e a excitação sexual, a capacidade de obter orgasmos, a capacidade de alcançar e manter uma ereção (para os homens) ou uma lubrificação (para as mulheres) e satisfação geral com a vida sexual. A atribuição das pontuações é feita de acordo com uma escala de tipo Likert de sete pontos: 1 = totalmente ausente; 2 = marcadamente diminuída; 3 = quase normal; 4 = normal; 5 = algo acima do normal;
6 = marcadamente acima do normal; e 7 = completamente acima do normal. As pontuações mais elevadas obtidas no MGH-SFQ indicam melhor funcionamento sexual. O seu reduzido número de itens faz com que esse instrumento seja de um enorme interesse para a prática clínica no momento de detetar disfunções sexuais, apresentou um excelente valor para a consistência interna (alfa $=.95)$.

Com o intuito de se avaliarem as medidas biomédicas e de traçar o perfil lipídico, foi usado o Accutrend ${ }^{\circledR}$ Plus (Roche Diagnostics $\mathrm{GmbH}$, Mannheim, Alemanha), um dispositivo portátil usado por técnicos de saúde para o diagnóstico da híperlipidemia, especificamente os seguintes parâmetros: colesterol total (CT) e triglicéridos (TG). 0 dispositivo determina as concentrações de lípidos a partir de amostras de sangue capilar a partir de duas gotas de sangue (15-40 $\mu \mathrm{L})$, usa uma lanceta aplicada aos dedos dos participantes. 0 dispositivo Accutrend ${ }^{\circledR}$ Plus é um teste serológico capilar que se baseia na retenção de células sanguíneas por filtração por meio de um velo de fibra de vidro quando se aplica uma gota de sangue numa tira reativa. A reação enzimática que acontece na zona subjacente requer uma quantidade adequada de oxigénio, o que resulta na formação de um produto de coloração oxidada. A reflectância da tira (medida a $660 \mathrm{~nm}$ ) é convertida em concentração por meio de um algoritmo. 0 ensaio de precisão interno do Accutrend ${ }^{\circledR}$ Plus determinado pelo fabricante é de $3,7 \%$ e $3,4 \%$ para o CT e TG respetivamente, ao passo que o ensaio de precisão externo com uma solução de controlo foi menor do que 5,0\% para o CT e 2,4\% para os TG. O tempo de prova é de 180 segundos $^{22,23}$. O rigor e a precisão do dispositivo Accutrend ${ }^{\circledR}$ Plus para as medidas de CT e TG têm sido avaliados por meio de estudos comparativos com medidas serológicas laboratoriais, verificou-se que as medidas de concentração obtidas por esse instrumento estão adequadamente correlacionadas com as medidas obtidas em laboratório, tornam-no num instrumento fiável e válido para medir os níveis de CT e TG.

Ao nível do perfil glicémico, pode-se salientar que a avaliação dos níveis de glicose no sangue capilar foi efetuada por meio do dispositivo OneTouch ${ }^{\circledR}$ Verio ${ }^{\circledR} \mathrm{IQ}$ (LifeScan ${ }^{\circledR}$ Johnson and Johnson, EUA). Esse instrumento permite a monitoração dos valores de glicémia no sangue, por meio de uma gota de $0,4 \mu \mathrm{L}$ obtida a partir de lanceta e que é sujeita a análise por meio de uma reação de GDH-FAD (glucose deshidrogenase com flavina-adenina dinucleótido) que ocorre numa tira com um biossensor enzimático. 0 dispositivo está calibrado para fornecer resultados correlacionados com o valor de glicose presente no plasma e é regulamentado pela legislação internacional que obriga à certificação pela International Organization for Standardization, norma ISO 15197, que, por sua vez, obriga a taxas de rigor na comparação com medidas de glicose laboratoriais superiores a $90 \%{ }^{24}$. O tempo de prova é de cinco segundos e os resultados de precisão com as medidas laboratoriais garantem que os resultados obtidos pelo dispositivo OneTouch ${ }^{\circledR}$ Verio ${ }^{\circledR}$ IQ são fiáveis e válidos para medir os níveis de glicémia no sangue.

Para a avaliação dos níveis de pressão arterial sistólica, diastólica, pulsação e arritmia foi usado o dispositivo de pressão sanguínea BMG $5610 \mathrm{AEG}^{\circledR}$, que permite medir a tensão arterial de forma automática e está calibrado de acordo com as medidas-padrão da Organização Mundial de Saúde (OMS), medem-se as seguintes amplitudes: 
sistólica - 60-260 mmHg; diastólica - 40-199 mmHg; pulsação - 40-180 batidas/minuto; e presença ou não de batimento cardíaco irregular.

Para se calcular o Índice de Massa Corporal (IMC), foi necessário medir a altura $(\mathrm{m})$ e o peso corporal $(\mathrm{Kg})$ do indivíduo, a fórmula usada foi a seguinte: $I M C=\mathrm{Kg} / \mathrm{m}^{2} 25$.

\section{Procedimento}

Após aprovação da Comissão de Ética da Faculdade de Ciências da Saúde da Universidade da Beira Interior (UBI Covilhã, Portugal), a amostra foi recolhida presencialmente nas instalações da UBI, sempre na presença de uma enfermeira que recolheu as amostras sorológicas e de um membro da equipa de investigação que monitorou a aplicação do inquérito.

Os participantes foram informados sobre a importância de se apresentar em jejum quando da recolha dos indicadores biomédicos e, posto isso, "picavam" o dedo para se recolher amostra de plasma e respetiva parametrização com os instrumentos de medida biomédica e seguidamente preenchiam um protocolo que incluía o questionário sociodemográfico e o Massachusetts General Hospital - Sexual Functioning Questionnaire (MGH-SFQ).

A aplicação dos questionários e a recolha dos indicadores biomédicos implicaram um período de tempo de cerca de 20 minutos por pessoa e foram feitas apenas num momento com cada indivíduo, em formato de autorresposta. Deu-se a possibilidade, a quem manifestasse esse interesse, de obter informações sobre os resultados gerais da investigação. A recolha da amostra foi feita em outubro e novembro de 2016 e janeiro de 2017.

Por último, os dados recolhidos foram traduzidos para uma linguagem em que fosse passível seu uso pelo programa SPSS ${ }^{\circledR}$ - versão 24.0, com o intuito de se proceder a análise estatística dos dados. A base de dados original era constituída por 362 participantes, mas após a aplicação dos critérios de exclusão (indivíduos fumadores e mulheres que tomam pílula) ficou reduzida a 225 participantes.

\section{Análise de dados}

O SPSS 24.0 (IBM, Chicago, IL) foi usado para analisar as os resultados. Para além das medidas descritivas básicas (média, desvio-padrão, frequências), foram calculados os coeficientes de correlação de Pearson (dado que os pressupostos da normalidade e da homogeneidade foram verificados) e, para a comparação de diferenças entre grupos, usou-se o $t$ de Student, para um nível de confiança de $95 \%$ $(p<0,05)$. Usou-se para determinar o efeito mediador das variáveis biomédicas sobre o funcionamento sexual a estimativa de modelos preditivos com base em regressão múltipla hierárquica. Os testes paramétricos foram usados por se verificarem os pressupostos para a normalidade, conforme apurado por meio do teste de Kolmogorov-Smirnov.

\section{Resultados}

Quando perguntados acerca do seu interesse sexual, $91(40,40 \%)$ participantes afirmaram ter interesse sexual normal, $52(23,10 \%)$ quase normal, 44 (19,60\%) marcadamente diminuído, $15(6,70 \%)$ totalmente ausente, $14(6,20 \%)$ algo acima do normal, oito $(3,60 \%)$ marcadamente acima do normal e apenas um $(0,40 \%)$ completamente acima do normal. Tendo em conta que a variável é suscetível a uma análise das médias, verificamos que a média é de 3,32 e o desvio-padrão de 1,19 , o que nos indica que, no geral, os participantes deste estudo apresentam o interesse sexual quase normal.

Relativamente à capacidade de obter estimulação ou excitação sexual, $41,80 \%$ da amostra $(n=94)$ referenciou como normal, $58(25,80 \%)$ quase normal, 38 (16,90\%) marcadamente diminuída, $15(6,70 \%)$ totalmente ausente, 11 $(4,90 \%)$ algo acima do normal, oito $(3,60 \%)$ marcadamente acima do normal e apenas um $(0,40 \%)$ completamente acima do normal. Tendo em conta a análise das médias, averiguamos que a média é de 3,34 e o desvio-padrão de 1,15, o que nos permite constatar que, no geral, os participantes deste estudo apresentam a capacidade de obter estimulação ou excitação sexual quase normal.

No que diz respeito à capacidade de obter orgasmos, verificamos que quase metade da amostra ( $n=100 ; 44,40 \%$ ) classificou a sua capacidade como normal, 50 (22,20\%) como quase normal, $42(18,70 \%)$ marcadamente diminuída, 19 $(8,40 \%)$ como totalmente ausente, sete $(3,10 \%)$ como algo acima do normal, cinco $(2,20 \%)$ marcadamente acima do normal e dois $(0,90 \%)$ como completamente acima do normal. Constatamos que a média de respostas a esse item é de 3,25 e o desvio-padrão de 1,17 , o que nos indica que, no geral, os participantes deste estudo apresentam a capacidade para obter orgasmos quase normal.

No que concerne à capacidade dos homens para atingir e manter ereção ou de as mulheres uma lubrificação, observamos na tabela 5 que 90 (40,00\%) participantes consideram a sua capacidade como normal, 56 (24,90\%) como quase normal, $39(17,30 \%)$ marcadamente diminuída, 21 (9,30\%) totalmente ausente, $12(5,30 \%)$ como algo acima do normal, quatro $(1,80 \%)$ como marcadamente acima do normal e três $(1,30 \%)$ como completamente acima do normal. Ao verificar que a média de respostas a esse item é de 3,25 e o desvio-padrão de 1,21, é possível afirmar que, no geral, os homens deste estudo apresentam a capacidade para atingir e manter ereção quase normal, assim como as mulheres uma lubrificação.

Finalmente, verificamos que, quando inquiridos sobre a satisfação com a sua vida sexual, $71(31,60 \%)$ participantes classificaram-na como normal, 65 (28,90\%) como quase normal, 42 (18,70\%) como marcadamente diminuída, 17 (7,60\%) como algo acima do normal, $14(6,20 \%)$ como totalmente ausente, $14(6,20 \%)$ como marcadamente acima do normal e dois $(0,90 \%)$ como completamente acima do normal. Ao constatar que a média de respostas a este item é de 3,38 e o desvio-padrão é de 1,27, averiguamos que, no geral, os participantes deste estudo apresentam uma satisfação com a sua vida sexual quase normal.

Foi, ainda, criada uma variável de funcionamento sexual total correspondente à média ponderada dos cinco itens constituintes da escala, em relação ao qual aferimos que o valor 20 ( 5 itens $\times 4$ pontos $=20$ ) seria o ponto de corte teórico normal e como tal verificamos que $59,6 \%(n=134)$ dos participantes apresentam um funcionamento sexual global que se encontra abaixo do ponto de corte teórico normal 
Tabela 1 Resultados para a comparação do funcionamento sexual e das variáveis biomédicas por género

\begin{tabular}{|c|c|c|c|c|c|}
\hline & Género & Média & $\begin{array}{l}\text { Desvio- } \\
\text { padrão }\end{array}$ & $t(d f)$ & $\mathrm{p}$ - valor \\
\hline \multirow[t]{2}{*}{ Interesse sexual } & Homem & 3,48 & 1,16 & $1,88(222)$ & 0,061 \\
\hline & Mulher & 3,18 & 1,20 & & \\
\hline \multirow{2}{*}{$\begin{array}{l}\text { Capacidade para obter } \\
\text { estimulação ou excitação } \\
\text { sexual }\end{array}$} & Homem & 3,46 & 1,07 & $1,53(222)$ & 0,128 \\
\hline & Mulher & 3,22 & 1,23 & & \\
\hline \multirow{2}{*}{$\begin{array}{l}\text { Capacidade para obter } \\
\text { orgasmos }\end{array}$} & Homem & 3,42 & 1,07 & $2,09(222)$ & $0,037^{*}$ \\
\hline & Mulher & 3,09 & 1,25 & & \\
\hline \multirow{2}{*}{$\begin{array}{l}\text { Capacidade de alcançar } \\
\text { e manter uma ereção } \\
\text { ou uma lubrificação }\end{array}$} & Homem & 3,37 & 1,18 & $1,41(222)$ & 0,161 \\
\hline & Mulher & 3,15 & 1,25 & & \\
\hline \multirow[t]{2}{*}{ Satisfação com a vida sexual } & Homem & 3,48 & 1,20 & $1,14(222)$ & 0,255 \\
\hline & Mulher & 3,28 & 1,34 & & \\
\hline \multirow[t]{2}{*}{ Glicémia } & Homem & 118,33 & 33,44 & $2,35(208)$ & $0,020^{*}$ \\
\hline & Mulher & 107,46 & 33,68 & & \\
\hline \multirow[t]{2}{*}{ Colesterol total } & Homem & 166,29 & 50,46 & $-1,66(210)$ & 0,098 \\
\hline & Mulher & 176,87 & 41,97 & & \\
\hline \multirow[t]{2}{*}{ Triglicéridos } & Homem & 143,96 & 65,58 & ,21 (191) & 0,831 \\
\hline & Mulher & 141,55 & 89,32 & & \\
\hline \multirow[t]{2}{*}{ Pressão sistólica } & Homem & 129,60 & 10,58 & 4,25 (197) & $0,000^{* *}$ \\
\hline & Mulher & 123,29 & 10,34 & & \\
\hline \multirow[t]{2}{*}{ Pressão diastólica } & Homem & 78,16 & 8,16 & 1,51 (195) & 0,132 \\
\hline & Mulher & 75,93 & 12,13 & & \\
\hline \multirow[t]{2}{*}{ Pulsação } & Homem & 75,40 & 11,04 &,$- 03(187)$ & 0,978 \\
\hline & Mulher & 75,44 & 10,18 & & \\
\hline \multirow[t]{2}{*}{ IMC } & Homem & 26,37 & 5,05 & $2,29(222)$ & $0,023^{*}$ \\
\hline & Mulher & 24,93 & 4,36 & & \\
\hline
\end{tabular}

e que apenas $40,4 \%(n=91)$ se encontram dentro do ponto de corte teórico normal ou apresentam valores superiores, o que nos permite constatar que, no geral, os participantes deste estudo apresentam um funcionamento sexual global abaixo dos valores esperados.

$\mathrm{Na}$ tabela 1 apresentam-se todos os valores para todas as medidas por género, assim como as respetivas significâncias estatísticas. Conforme se pode verificar, os homens apresentam, em média, melhor funcionamento sexual global do que as mulheres, assim como nos valores da glicémia, triglicéridos, pressão sistólica e pressão diastólica e IMC. Por sua vez, as mulheres apresentam médias superiores no colesterol total e na pulsação (tabela 2).

Para melhor compreensão dos resultados em relação à idade e tendo em conta que a média de idades da amostra é de 40,53 anos, optou-se por fazer subgrupos, para tal dividiu-se a amostra em duas partes iguais, uma constituída pelos participantes mais novos (até aos 40 anos) e outra pelos participantes mais velhos (a partir dos 40 anos). Quando analisamos a tabela 1, apuramos que o grupo dos mais novos apresenta, em média, melhor funcionamento sexual quando comparado com o grupo dos mais velhos. No que toca às variáveis biomédicas, é de realçar que só ao nível da pulsação é que o grupo dos mais novos apresenta uma média superior, quando comparado com o grupo dos mais velhos. Nas restantes variáveis biomédicas é o grupo dos mais velhos que apresenta uma média superior.

Da avaliação do grau de associação entre o funcionamento sexual global e as variáveis biomédicas obtiveram-se coeficientes de correlação estatisticamente significativos para o IMC ( $r=-0,253 ; p<0,001)$, glicémia $(r=-0,230$; $p<0,001)$ e colesterol total $(r=-0,144 ; p<0,05)$, que nos indicam que quanto mais funcionamento sexual global o participante tem, menos IMC, menos glicémia e menos colesterol total vai apresentar. Não se obtiveram valores estatisticamente significativos para os triglicéridos $(r=$ $-0,067)$, a pressão sistólica $(r=0,004)$, a pressão diastólica $(r=0,040)$ e para a pulsação $(r=-0,049)$. Os coeficientes de correlação obtidos para todas as outras variáveis podem ser encontrados na tabela 3.

Por meio da tabela 4 é possível estimar a modulação e determinar o efeito mediador das variáveis biomédicas sobre o funcionamento sexual. Constata-se que se só existisse um modelo preditivo a explicar o bom funcionamento sexual, tendo em conta só com base nas variáveis sociodemográficas, ele só explicaria $23 \%$ da variância $\left(r^{2}=0,23\right.$; $p<0,001)$. Contudo, quando se acrescentam as variáveis biomédicas o modelo já passa a explicar $31 \%\left(r^{2}=0,31 ; p<\right.$ $0,001)$. Salienta-se que, desse modo, o modelo explica $31 \%$ da variância total. No passo 1 , constata-se que a idade tem 
Tabela 2 Resultados para a comparação do funcionamento sexual e das variáveis biomédicas tendo em conta a faixa etária

\begin{tabular}{|c|c|c|c|c|c|}
\hline & Faixa etária & Média & $\begin{array}{l}\text { Desvio- } \\
\text { padrão }\end{array}$ & $t(d f)$ & $\mathrm{p}$ - valor \\
\hline \multirow[t]{2}{*}{ Interesse sexual } & $<40$ & 3,75 & 1,23 & $5,28(210,00)$ & $0,000^{* *}$ \\
\hline & $>41$ & 2,93 & 1,00 & & \\
\hline \multirow{2}{*}{$\begin{array}{l}\text { Capacidade para obter } \\
\text { estimulação ou excitação } \\
\text { sexual }\end{array}$} & $<40$ & 3,74 & 1,18 & $5,43(210,00)$ & $0,000^{* * *}$ \\
\hline & $>41$ & 2,93 & ,96 & & \\
\hline \multirow{2}{*}{$\begin{array}{l}\text { Capacidade para obter } \\
\text { orgasmos }\end{array}$} & $<40$ & 3,58 & 1,23 & $4,56(210,00)$ & $0,000^{* * *}$ \\
\hline & $>41$ & 2,89 & ,99 & & \\
\hline \multirow{2}{*}{$\begin{array}{l}\text { Capacidade de alcançar e } \\
\text { manter uma ereção ou uma } \\
\text { lubrificação }\end{array}$} & $<40$ & 3,65 & 1,31 & $5,04(191,60)$ & $0,000^{* *}$ \\
\hline & $>41$ & 2,86 & ,95 & & \\
\hline \multirow[t]{2}{*}{ Satisfação com a vida sexual } & $<40$ & 3,80 & 1,33 & $5,60(195,56)$ & $0,000^{* * *}$ \\
\hline & $>41$ & 2,90 & 1,00 & & \\
\hline \multirow[t]{2}{*}{ Glicémia } & $<40$ & 101,70 & 23,25 & $-5,04(158,86)$ & $0,000^{* *}$ \\
\hline & $>41$ & 124,54 & 38,89 & & \\
\hline \multirow[t]{2}{*}{ Colesterol total } & $<40$ & 154,31 & 36,26 & $-5,23(188,75)$ &, $000^{* *}$ \\
\hline & $>41$ & 186,48 & 50,52 & & \\
\hline \multirow[t]{2}{*}{ Triglicéridos } & $<40$ & 131,06 & 82,21 & $-2,16(186,00)$ & $0,032^{*}$ \\
\hline & $>41$ & 155,51 & 72,92 & & \\
\hline \multirow[t]{2}{*}{ Pressão sistólica } & $<40$ & 124,78 & 10,44 & $-1,93(189,00)$ & 0,055 \\
\hline & $>41$ & 127,85 & 11,47 & & \\
\hline \multirow[t]{2}{*}{ Pressão diastólica } & $<40$ & 74,92 & 7,30 & $-2,73(187,00)$ & $0,007^{*}$ \\
\hline & $>41$ & 79,03 & 12,63 & & \\
\hline \multirow[t]{2}{*}{ Pulsação } & $<40$ & 76,02 & 12,98 & ,71 $(144,69)$ & 0,480 \\
\hline & $>41$ & 74,90 & 7,72 & & \\
\hline \multirow[t]{2}{*}{ IMC } & $<40$ & 24,00 & 4,24 & $-5,47(209,98)$ & $0,000^{* *}$ \\
\hline & $>41$ & 27,17 & 4,19 & & \\
\hline
\end{tabular}

${ }^{*}<0,05$.

$* *<0,001$.

Tabela 3 Matriz de correlação de fatores do funcionamento sexual e de variáveis biomédicas

\begin{tabular}{|c|c|c|c|c|c|c|c|c|c|c|c|c|c|}
\hline & MGH 1 & MGH 2 & MGH 3 & MGH 4 & MGH 5 & MGH total & IMC & Glic. & $\begin{array}{l}\text { Colest. } \\
\text { total }\end{array}$ & Trigli. & $\begin{array}{l}\text { Pres. } \\
\text { sist. }\end{array}$ & $\begin{array}{l}\text { Pres. } \\
\text { diast. }\end{array}$ & Puls. \\
\hline MGH 1 & 1 & & & & & & & & & & & & \\
\hline MGH 2 & $0,888^{* *}$ & 1 & & & & & & & & & & & \\
\hline MGH 3 & $0,813^{* *}$ & $0,886^{* *}$ & 1 & & & & & & & & & & \\
\hline MGH 4 & $0,757^{* *}$ & $0,824^{* *}$ & $0,813^{* *}$ & 1 & & & & & & & & & \\
\hline MGH 5 & $0,690^{* *}$ & $0,749^{* *}$ & $0,749^{* *}$ & $0,729^{* *}$ & 1 & & & & & & & & \\
\hline MGH total & $0,908^{* *}$ & $0,951^{* *}$ & $0,933^{* *}$ & $0,905^{* *}$ & $0,863^{* *}$ & 1 & & & & & & & \\
\hline IMC & $-0,234^{* *}$ & $-0,253^{* * *}$ & $-0,236^{* *}$ & $-0,221^{\text {** }}$ & $-0,211^{\text {** }}$ & $-0,253^{* *}$ & 1 & & & & & & \\
\hline Glicémia & $-0,181^{* *}$ & $-0,221^{* *}$ & $-0,209^{* *}$ & $-0,221^{* *}$ & $-0,220^{* *}$ & $-0,230^{* *}$ & $0,457^{* *}$ & 1 & & & & & \\
\hline $\begin{array}{l}\text { Colesterol } \\
\text { total }\end{array}$ & $-0,165^{*}$ & $-0,141^{*}$ & $-0,093$ & $-0,080$ & $-0,173^{*}$ & $-0,144^{*}$ & $0,450^{* * *}$ & $0,590^{* *}$ & 1 & & & & \\
\hline Triglicéridos & $-0,034$ & $-0,050$ & $-0,052$ & $-0,020$ & $-0,149^{*}$ & $-0,067$ & $0,377^{* *}$ & $0,503^{* * *}$ & $0,507^{* *}$ & 1 & & & \\
\hline $\begin{array}{l}\text { Pressão } \\
\text { sistólica }\end{array}$ & 0,000 & $-0,015$ & $-0,027$ & 0,006 & 0,050 & 0,004 & $0,379^{* * *}$ & $0,398^{* *}$ & 0,135 & $0,237^{* *}$ & 1 & & \\
\hline $\begin{array}{l}\text { Pressão } \\
\text { diastólica }\end{array}$ & $-0,020$ & 0,051 & 0,053 & 0,055 & 0,043 & 0,040 & $0,386^{* *}$ & $0,455^{* *}$ & $0,382^{* *}$ & $0,307^{* *}$ & 0,556 & 1 & \\
\hline Pulsação & $-0,061$ & $-0,037$ & $-0,044$ & $-0,002$ & $-0,079$ & $-0,049$ & 0,081 & 0,125 & $0,157^{*}$ & 0,115 & $-0,019$ & ,230** & 1 \\
\hline
\end{tabular}


Tabela 4 Resultados para o modelo de regressão múltipla hierárquica preditivo do funcionamento sexual global

\begin{tabular}{llll}
\hline Preditores & $\mathrm{r}^{2}$ & $\beta$ & $\mathrm{p}$ \\
\hline Passo 1 & 0,233 & & $0,000^{* *}$ \\
Idade & & $-0,464$ & $0,000^{* * *}$ \\
Sexo & & $-0,110$ & 0,118 \\
Orientação sexual & & 0,057 & 0,412 \\
Passo 2 & 0,305 & & $0,000^{* * *}$ \\
Idade & & $-0,382$ & $0,000^{* *}$ \\
Sexo & & $-0,128$ & 0,073 \\
Orientação sexual & & 0,089 & 0,280 \\
Glicémia & & $-0,089$ & 0,423 \\
Colesterol total & & 0,119 & 0,293 \\
Triglicéridos & & 0,090 & 0,302 \\
Pressão sistólica & & 0,044 & 0,627 \\
Pressão diastólica & & 0,136 & 0,147 \\
Pulsação & $-0,084$ & 0,243 \\
IMC & & $-0,303$ & $0,002^{*}$ \\
\hline * <0,05. & & \\
** 0,001. & & & \\
& & &
\end{tabular}

correlação negativa $(\beta=-0,46 ; p<0,001)$. Portanto, quanto maior for a idade, menor será o funcionamento sexual. No passo 2 , salienta-se que a idade $(\beta=-0,38 ; p<0,001)$ e 0 IMC $(\beta=-0,30 ; p<0,05)$ são as variáveis que têm maior poder explicativo e preditivo.

\section{Discussão}

Nesta investigação procurou-se desenvolver procedimentos metodológicos para se compreender e explicar a interferência que os indicadores biomédicos têm no funcionamento sexual, começou-se por entender as diferenças entre homens e mulheres e faixas etárias.

Assim, relativamente ao género, é de destacar que os homens pontuam em todos os domínios do funcionamento sexual uma média superior quando comparados com as mulheres, logo é o sexo masculino que apresenta melhores níveis de funcionamento sexual. Esses resultados são congruentes com aquilo que a literatura tem sustentado ao longo de várias décadas ${ }^{8,11,26-28}$. É importante salientar que os fatores biológicos influenciam o desenvolvimento sexual do homem, acabam por afetar o desejo, o funcionamento sexual e, de um modo indireto, a satisfação sexual, ao passo que se racionalizou a sexualidade feminina, tornou-se-a mais suscetível às influências culturais e transformou-se o corpo feminino num objeto mensurável e que é sujeito a várias práticas de objetivação. A mulher ainda tem como funções sociais o casamento, a maternidade e a educação dos filhos e é esse papel imposto pela sociedade às mulheres que as incita a controlarem os seus pensamentos, de modo a que não saiam das normas socialmente impostas, o que, por seu turno, afeta os seus mecanismos de expressão sexual 29. Pode-se, assim, afirmar que as influências históricoculturais do nosso contexto podem ter-se refletido no modo das mulheres pensarem e agirem sobre o seu funcionamento sexual e, consequentemente, isso ter afetado as suas respostas.
No nosso estudo também se constata que relativamente à idade são os participantes mais novos (até aos 40 anos) que apresentam melhor funcionamento sexual em todos os seus domínios e também são esses participantes que apresentam níveis médios mais baixos nos indicadores biomédicos, ou seja, melhores níveis para a saúde. Esses dados vão de encontro com o que seria esperado, pois, segundo a literatura, a disfunção sexual está diretamente associada com o aumento da idade ${ }^{30-33}$, as pessoas mais velhas apresentam falta de interesse em sexo, incapacidade em obter orgasmos e os homens apresentam mais dificuldades ao nível da ereção ${ }^{34}$. Um dos fatores que podem contribuir para o aumento da disfunção sexual com o aumento da idade é a redução dos níveis de testosterona ao longo dos anos e o aumento de ocorrência doença e toma de medicação, mas também a associação do preconceito e estigma sexual em relação à idade ${ }^{35}$.

Da análise das correlações, destaca-se que o aumento do IMC, da glicémia, do colesterol total, dos triglicéridos e da pulsação está associado à diminuição do funcionamento sexual global e todos os seus domínios. Esses resultados são congruentes com os de outros estudos que salientam que a disfunção sexual está diretamente relacionada com o IMC ${ }^{13,15}$, com o diabetes ${ }^{13,14}$ e com os altos níveis de colesterol e triglicéridos ${ }^{13,16}$. Esses fatores parecem estar associados ao facto de, quando muito elevados, o açúcar e os lípidos no sangue poderem criar placas nas artérias, interferir no fluxo sanguíneo necessário para o adequado funcionamento sexual.

Fica claro, portanto, que as variáveis biomédicas condicionam a resposta sexual humana, esses resultados têm implicação para a intervenção na área da saúde, visto que a investigação sobre a relação que existe entre as variáveis biomédicas e o funcionamento sexual pode ser uma ferramenta útil para identificar pacientes que estejam em risco devido a alguma doença (por exemplo, a doença cardiovascular) que ainda não se tenha manifestado por outros sintomas ou sinais, pois e de acordo com diversos estudos pacientes com disfunções sexuais apresentam uma alta incidência de doenças cardiovasculares ${ }^{17,18}$ e hipertensão arterial $^{19}$.

Este estudo não é isento de limitações, pois o facto de a amostra ter sido recolhida por inquérito pode desencadear o efeito de desejabilidade social, as condições de recolha da amostra e o facto de essa amostra ser por conveniência são os principais inconvenientes a apontar. Contudo, esta investigação também apresenta vantagens, como o facto de preencher lacunas que existem e o facto de a amostra ser extremamente controlada (sem indivíduos que fumem e/ou tomem pílula).

Ainda existe uma clara necessidade de se fazerem mais estudos, especialmente estudos longitudinais, para se obterem dados mais precisos sobre a funcionalidade sexual em homens e mulheres. Esperamos, com esta investigação, evidenciar a importância dos indicadores biomédicos na expressão da funcionalidade sexual, na medida em que os técnicos que trabalhem com adultos sexualmente ativos deverão estar atentos ao modo como as variáveis biomédicas interferem na expressão sexual. Fica, também, claro que existem problemas de natureza sexual que tornam necessárias intervenções específicas, nomeadamente para 
as pessoas mais velhas e para as mulheres, de maneira a melhorar o seu funcionamento sexual, enfatiza-se o modo como as variáveis estudadas constituem uma peça-chave na melhoria dos seus indicadores de saúde sexual.

Finalmente, acreditamos que os resultados produzidos por esta investigação poderão ser aplicados á discussáo conceptual dos direitos sexuais em adultos saudáveis, particularmente na área da sexologia, preencher também uma lacuna de investigação sistemática nessa área em Portugal.

\section{Responsabilidades éticas}

Direito à privacidade e consentimento escrito. Os autores declaram ter recebido consentimento escrito dos sujeitos mencionados no artigo.

Confidencialidade dos dados. Os autores declaram que não aparecem dados de participantes neste artigo.

Proteção de pessoas e animais. Os autores declaram que para esta investigação não se fizeram experiências em seres humanos e/ou animais.

\section{Conflitos de interesse}

Os autores declaram não haver conflitos de interesse.

\section{Referências}

1. Diamond L, Huebner D. Is good sex good for you? Rethinking sexuality and health. Social Personality Psych Compass. 2012;6:54-69.

2. McCarthy B, Wald L. Mindfulness and good enough sex. Sex Relationship Therapy. 2013;28(1-2):39-47.

3. Frohlich P, Meston C. Sexual functioning and self-reported depressive symptoms among college women. J Sex Research. 2002;39:321-5.

4. Marchal-Bertrand L, Espada J, Morales A, Gómez-Lugo M, Soler F, Vallejo-Medina P. Adaptation, validation, and reliability of the Massachusetts General Hospital-Sexual Functioning Questionnaire in a Colombian sample and factorial equivalence with the Spanish version. Rev Latinoamericana Psicología. 2016;48:88-97.

5. DeRogatis L, Burnett A. The epidemiology of sexual dysfunctions. J Sex Medicine. 2008;5:289-300.

6. Laumann E, West S, Glasser D, Carson C, Rosen R, Kang J. Prevalence and correlates of erectile dysfunction by race and ethnicity among men aged 40 or older in the United States: from the Male Attitudes Regarding Sexual Health Survey. J Sex Medicine. 2011;4:57-65.

7. Lewis R. Epidemiology of sexual dysfunction in Asia compared to the rest of the world. Asian Journal of Andrology. 2010;13:152-8.

8. Lewis R, Fugl-Meyer K, Bosch R, Fugl-Meyer A, Laumann E, Lizza E, et al. Epidemiology/risk factors of sexual dysfunction. J Sex Medicine. 2004;1:35-9.

9. Lewis R, Fugl-Meyer K, Corona G, Hayes R, Laumann E, Moreira $E$, et al. Definitions/epidemiology/risk factors for sexual dysfunction. J Sex Medicine. 2010;7:1598-607.

10. Nicolosi A, Glasser DB, Kim SC, Marumo K, Laumann EO, Group $\mathrm{GI}$. Sexual behaviour and dysfunction and help-seeking patterns in adults aged $40-80$ years in the urban population of Asian countries. BJU Int. 2005;95:609-14.
11. Nicolosi A, Laumann E, Glasser D, Moreira E, Paik A, Gingell C. Sexual behavior and sexual dysfunctions after age 40: The global study of sexual attitudes and behaviors. Urology. 2004;64:991-7.

12. Vendeira PS, Pereira NM, Tomada N, Carvalho JL, Episex-PT:. Epidemiologia das disfunções sexuais masculinas em Portugal. ISEX Cadernos de Sexologia. 2011;4:15-22.

13. Wang G, Wang L, Wang Y, Li M. Risk factors for sexual dysfunction among Chinese women with type 2 diabetes. Inter J Diabetes in Developing Countries. 2014;35:219-24.

14. Kaya Erten Z, Zincir H, Özkan F, Selçuk A, Elmali F. Sexual lives of women with diabetes mellitus (type 2 ) and impact of culture on solution for problems related to sexual life. J Clinical Nursing. 2013;23(7-8):995-1004.

15. Pinnock CB, Stapleton AM, Marshall VR. Erectile dysfunction in the community: a prevalence study. Med J. 1999;171: 353-7.

16. Fung $M$, Bettencourt R, Barrett-Connor E. Heart disease risk factors predict erectile dysfunction 25 years later. J American College of Cardiology. 2004;43:1405-11.

17. Anyfanti P, Pyrpasopoulou A, Triantafyllou A, Doumas M, Gavriilaki E, Triantafyllou G, et al. The impact of frequently encountered cardiovascular risk factors on sexual dysfunction in rheumatic disorders. Andrology. 2013;1: 556-62.

18. Stein R, Sardinha A, Araújo C. Sexual activity and heart patients: a contemporary perspective. Canadian J Cardiology. 2016;32:410-20.

19. Nascimento E, Maia A, Nardi A, Silva A. Sexual dysfunction in arterial hypertension women: the role of depression and anxiety. J Affective Disorders. 2015;181:96-100.

20. Labbate LA, Lare SB. Sexual dysfunction in male psychiatric outpatients: validity of the Massachusetts General Hospital Sexual Functioning Questionnaire. Psychother Psychosom. 2001;70:221-5.

21. Pereira H. Validação psicométrica da versão portuguesa do Massachusetts General Hospital - Sexual Functioning Questionnaire. Rev Internacional Andrologia. Aceite para publicação.

22. Scafoglieri A, Tresignie J, Provyn S, Clarys JP, Bautmans I. Reproducibility, accuracy and concordance of Accutrend ${ }^{\circledR}$ Plus for measuring circulating lipid concentration in adults. Biochemia Medica. 2012;22:100-8.

23. Coqueiro RDS, Santos MC, Neto JDSL, Queiroz BMD, Brügger NAJ, Barbosa AR. Validity of a portable glucose, total cholesterol, and triglycerides multi-analyzer in adults. Bio Res Nursing. 2013;16:288-94.

24. Ramchandani N, Heptulla RA. New technologies for diabetes: a review of the present and the future. Inter J Pediatric Endocrinology. 2011:1-10.

25. Moraes W, Poyares D, Zalcman I, De Mello MT, Bittencourt LR, Santos-Silva R, et al. Association between body mass index and sleep duration assessed by objective methods in a representative sample of the adult population. Sleep Med. 2013;14: 312-8.

26. Carpenter LM, Nathanson CA, Kim YJ. Physical women, emotional men: gender and sexual satisfaction in midlife. Archiv Sex Behav. 2009;38:87-107.

27. Laumann EO, Paik A, Rosen RC. Sexual dysfunction in the United States: prevalence and predictors. Jama. 1999;281: 537-44.

28. Pfeiffer E, Verwoerdt A, Davis GC. Sexual behavior in middle life. American Journal of Psychiatry. 1972;128:1262-7.

29. Martins APV. Visões do feminino: a medicina da mulher nos séculos XIX e XX. SciELO-Editora Fiocruz. 2004.

30. Bancroft J, Loftus J, Long JS. Distress about sex: a national survey of women in heterosexual relationships. Archiv Sex Behav. 2003;32:193-208. 
+Model

ANDROL-186; No. of Pages 9

31. DeLamater JD, Sill M. Sexual desire in later life. J Sex Res. 2005;42:138-49.

32. Oksuz E, Malhan S. Prevalence and risk factors for female sexual dysfunction in Turkish women. J Urol. 2006;175:654-8.

33. Saigal CS, Wessells H, Pace J, Schonlau M, Wilt TJ. Predictors and prevalence of erectile dysfunction in a racially diverse population. Archiv Internal Med. 2006;166:207-12.
34. Laumann EO, Nicolosi A, Glasser DB, Paik A, Gingell C, Moreira $E$, et al. Sexual problems among women and men aged 40-80 y: prevalence and correlates identified in the Global Study of Sexual Attitudes and Behaviors. Inter J Impotence Res. 2005; $17: 39$.

35. Siqueira TCB, Pereira ABM. Terceira idade e sexualidade: um encontro possível? Fragmentos de Cultura. 2007;17:271-7. 